\title{
Evidence of a truncated spectrum in the angular correlation function of the cosmic microwave background
}

\author{
F. Melia ${ }^{1}$ and M. López-Corredoira ${ }^{2,3}$
}

\author{
${ }^{1}$ Department of Physics, The Applied Math Program, and Department of Astronomy, The University of Arizona, AZ 85721, USA \\ e-mail: melia@physics . arizona.edu \\ 2 Instituto de Astrofísica de Canarias, 38205 La Laguna, Tenerife, Spain \\ ${ }^{3}$ Departamento de Astrofísica, Universidad de La Laguna, 38206 La Laguna, Tenerife, Spain
}

Received 26 October 2017 / Accepted 21 December 2017

\begin{abstract}
Aims. The lack of large-angle correlations in the fluctuations of the cosmic microwave background (CMB) conflicts with predictions of slow-roll inflation. But while probabilities $(\lesssim 0.24 \%)$ for the missing correlations disfavour the conventional picture at $\gtrsim 3 \sigma$, factors not associated with the model itself may be contributing to the tension. Here we aim to show that the absence of large-angle correlations is best explained with the introduction of a non-zero minimum wave number $k_{\min }$ for the fluctuation power spectrum $P(k)$.

Methods. We assumed that quantum fluctuations were generated in the early Universe with a well-defined power spectrum $P(k)$, although with a cut-off $k_{\min } \neq 0$. We then re-calculated the angular correlation function of the CMB and compared it with Planck observations.

Results. The Planck 2013 data rule out a zero $k_{\min }$ at a confidence level exceeding $8 \sigma$. Whereas purely slow-roll inflation would have stretched all fluctuations beyond the horizon, producing a $P(k)$ with $k_{\min }=0$ - and therefore strong correlations at all angles $-\mathrm{a}$ $k_{\min } \neq 0$ would signal the presence of a maximum wavelength at the time $\left(t_{\mathrm{dec}}\right)$ of decoupling. This argues against the basic inflationary paradigm, and perhaps even suggests non-inflationary alternatives, for the origin and growth of perturbations in the early Universe. In at least one competing cosmology, the $R_{\mathrm{h}}=c t$ universe, the inferred $k_{\min }$ corresponds to the gravitational radius at $t_{\mathrm{dec}}$.
\end{abstract}

Key words. cosmic background radiation - cosmology: observations - cosmology: theory - early Universe - inflation - large-scale structure of Universe

\section{Introduction}

The Wilkinson Microwave Anisotropy Probe (WMAP; Bennett et al. 2003; Spergel et al. 2003) and Planck (Planck Collaboration XII 2014) have resoundingly confirmed the existence of several anomalies seen on very large scales by the Cosmic Background Explorer (COBE; Wright et al. 1996; Hinshaw et al. 1996). The most prominent among these is the lack of any significant correlation measured at angles $\gtrsim 60^{\circ}$. Its possible impact on the basic inflationary paradigm (Guth 1981; Linde 1982) has spurred a prolonged debate concerning whether it is due to a real physical phenomenon or unrecognized observational systematic effects.

The absence of large-angle correlations may simply be due to cosmic variance (Bennett et al. 2013; Copi et al. 2015), although probabilities for the missing correlations are typically $\lesssim 0.24 \%$, disfavouring the basic inflationary picture at better than $3 \sigma$ (see also Kim \& Naselsky 2011; Melia 2014; Gruppuso et al. 2016). This anomaly may also be due to subtleties in the foreground subtraction or unrecognized instrumental systematics (Bennett et al. 2013), but this is far from settled.

The absence of large-angle correlations in the high-fidelity cosmic microwave background (CMB) maps poses one of the most serious challenges to the basic inflationary paradigm and, with it, to the internal self-consistency of the standard model. Since no theoretical corrections can improve the fit (Planck Collaboration XII 2014), the largest angular scales are probing different physics than the anisotropies seen at angles smaller than $\sim 2^{\circ}$ which, in contrast, are highly consistent with the predictions of the standard model.

In parallel with this dichotomy between the small- and largeangle correlations, WMAP and Planck have also revealed an unexpectedly weak power in the low- $\ell$ multipoles (see Eq. (6) below) compared with the corresponding power at higher $\ell$ 's (see e.g. Bennett et al. 2011). This anomaly may or may not be related to the absence of angular correlation at angles $\geq 60^{\circ}$ (Copi et al. 2007, 2015). Arguments have been made on both sides, although, if unrelated, the existence of two such anomalies significantly exacerbates the tension with the predictions of standard inflationary cosmology (see also Efstathiou 2004). The power deficit at large angular scales manifests itself in several ways, however, not just via these two particular facets, so its impact on the interpretation of the CMB fluctuations is extensive. A thorough consideration of the broader issues associated with the low angular power may be found in several recent publications by the Planck Collaboration XXIII (2014) and Planck Collaboration XVI (2016). In this paper, our focus is specifically on the interpretation of the angular correlation function, which may now be studied at an unprecedented level of precision.

Previous attempts at modifying the basic inflationary paradigm to address this issue have relied on the inflaton field evolving through an early fast-rolling stage, producing a characteristic scale when the wave number associated with the transition from fast to slow roll exited the horizon (Contaldi et al. 2003; Destri et al. 2010; Gruppuso et al. 2016). However, given 
the relative imprecision of the data available then (compared to the exquisite measurements provided most recently by Planck), the existence of such a scale could be established at no more than $\sim 2 \sigma$.

In one such attempt to determine whether the power spectrum is truncated, Niarchou et al. (2004) adopted a functional form (first introduced by Contaldi et al. 2003)

$P(k)=P_{0}(k)\left[1-\mathrm{e}^{-\left(k / k_{\mathrm{c}}\right)^{\alpha}}\right]$,

where

$P_{0}(k)=A k^{n}$,

is the usual primordial power-law spectrum, $k_{\mathrm{c}}$ is a characteristic wave number, and $\alpha=1.8$, and carried out a Bayesian model comparison based on the CMB power spectrum itself (rather than the angular correlation function) to demonstrate that the WMAP data available at that time preferred an attenuated $P(k)$ with $k_{\mathrm{c}} \approx(5-6) \times 10^{-4} \mathrm{Mpc}^{-1}$. If the last scattering surface occurred at $z_{\mathrm{cmb}} \sim 1080$ (according to the standard model), for which the expansion factor in a flat Universe was $a\left(z_{\mathrm{cmb}}\right)=1 /\left(1+z_{\mathrm{cmb}}\right) \approx 9.25 \times 10^{-4}$, this characteristic wave number would have corresponded to a physical fluctuation size $\lambda_{\max } \sim 10 \mathrm{Mpc}$. But for reasons we describe shortly, this use of the entire spectrum does not emphasize the large-angle anomalies, and Niarchou et al. (2004) concluded that a cut-off model such as Eq. (1) is preferred over one without a $k_{\mathrm{c}}$ at only the $\sim 1.4 \sigma$ level.

In this paper, we address the observed lack of angular correlation at large angles with a clear, unobstructed focus on the possible existence of a cut-off in the fluctuation spectrum as seen primarily at angles $\gg 1^{\circ}$, bolstered by the unprecedented accuracy of the Planck 2013 measurements. This distinctly different approach to the resolution of this anomaly avoids an unnecessary reliance on inflation, which may or may not have actually happened. A clear emergence of a non-zero $k_{\min }$ in the Planck data would motivate the search for new physics in both inflationary and non-inflationary scenarios. We note that the horizon problem plaguing $\Lambda$ cold dark matter (CDM) manifests itself only in models with an early period of deceleration, so inflation is not required in all Friedmann-Robertson-Walker cosmologies. For example, it is not present in models, such as the $R_{\mathrm{h}}=c t$ universe (Melia 2007, 2016, 2017; Melia \& Shevchuk 2012), in which opposite sides of the sky reached thermal equilibrium following the Big Bang (Melia 2013). In this paper, we seek to uncover compelling evidence in favour of such new physics beyond conventional, slow-roll inflation.

\section{Theoretical background}

To implement the introduction of a $k_{\min }$, we assume that quantum fluctuations were generated in the early Universe with a welldefined power spectrum $P(k)$, and that these seeds subsequently grew linearly towards $t_{\mathrm{dec}}$. But unlike the conventional picture, we find that the most important property of $P(k)$ that alleviates the anomaly is a non-zero value of the minimum wave number $k_{\text {min }}$, possibly due to an early transition from fast to slow roll, or generic to a variety of non-inflationary scenarios. The $R_{\mathrm{h}}=$ ct universe, which has been studied extensively in recent years, meets these criteria, therefore we know of at least one alternative cosmology with the necessary characteristics (Melia 2016).

In the current $\Lambda \mathrm{CDM}$, the fluctuations would have grown to all observable scales as a result of the rapid expansion during

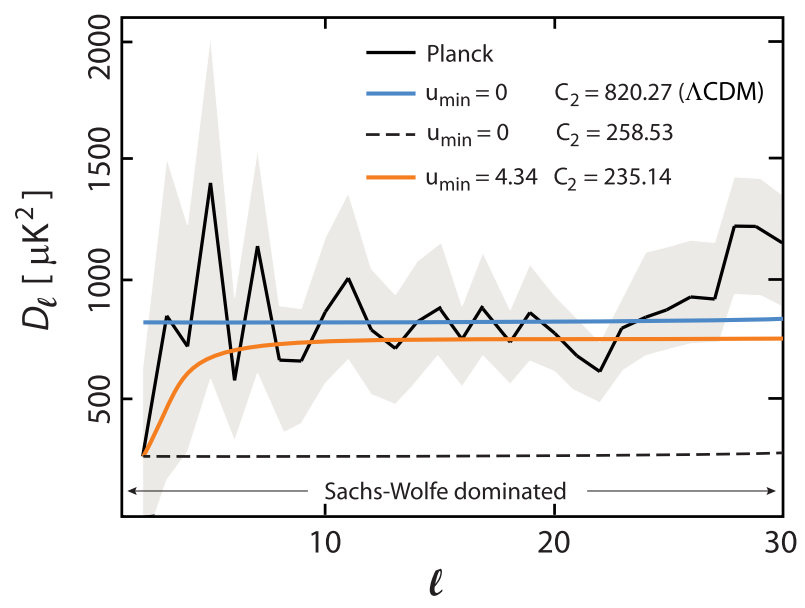

Fig. 1. Power spectrum (black) estimated with the NILC method (Planck Collaboration XII 2014), with $1 \sigma$ Fisher errors (grey shaded). The Planck $\Lambda$ CDM best fit model, including solely the Sachs-Wolfe (SW) effect, is shown in blue. The red curve for $u_{\text {min }} \neq 0$, and the dashed curve for $u_{\min }=0$, also based solely on SW, are optimized based on the best fit to $C(\cos \theta)$ shown in Fig. 2. The SW effect dominates at large angles $\gg 1-2^{\circ}$, corresponding to $\ell \lesssim 30$, while local physical effects, e.g. BAO, dominate for $\ell \gtrsim 30$.

inflation, hence $k_{\min }=0$. In contrast, a non-inflationary expansion would have had $k_{\min } \neq 0$ if the fluctuation growth was restricted to a finite range of wavelengths. Again resorting to $R_{\mathrm{h}}=c t$ as an example, $k_{\min }$ could have corresponded to $2 \pi$ times the Hubble radius $R_{\mathrm{h}}\left(t_{\mathrm{dec}}\right)$ at $t_{\mathrm{dec}}$. A similar correspondence would have applied to the formation of structure from topological defects (Brandenberger 1994). The results of our analysis should be independent of any specific model and relevant to any modified mechanism of inflation or to any non-inflationary cosmology that possesses a non-zero minimum wave number in its power spectrum $P(k)$.

We follow convention and assume that a Gaussian random field in the plane of the sky describes the microwave temperature $T(\hat{\mathbf{n}})$ in every direction $\hat{\mathbf{n}}$, and write it as an expansion in spherical harmonics $Y_{l m}(\hat{\mathbf{n}})$. The corresponding random coefficients $a_{l m}$ have zero mean, hence the angular correlation function linking directions $\hat{\mathbf{n}}_{1}$ and $\hat{\mathbf{n}}_{2}$ depends only on $\cos \theta \equiv \hat{\mathbf{n}}_{1} \cdot \hat{\mathbf{n}}_{2}$, which we expand in terms of Legendre polynomials as follows:

$C(\cos \theta) \equiv\left\langle T\left(\hat{\mathbf{n}}_{1}\right) T\left(\hat{\mathbf{n}}_{2}\right)\right\rangle=\frac{1}{4 \pi} \sum_{\ell}(2 \ell+1) C_{\ell} P_{\ell}(\cos \theta)$.

The coefficients are statistically independent, therefore

$\left\langle a_{\ell m}^{*} a_{\ell^{\prime} m^{\prime}}\right\rangle \propto \delta_{\ell \ell^{\prime}} \delta_{m m^{\prime}}$,

and statistical isotropy guarantees that the constant of proportionality depends only on $\ell$,

$\left\langle a_{\ell m}^{*} a_{\ell^{\prime} m^{\prime}}\right\rangle=C_{\ell} \delta_{\ell \ell^{\prime}} \delta_{m m^{\prime}}$.

The expansion coefficient in Eq. (3),

$C_{\ell}=\frac{1}{2 \ell+1} \sum_{m}\left|a_{\ell m}\right|^{2}$,

is known as the angular power of multipole $\ell$.

In the ideal case of full-sky coverage, $C(\cos \theta)$ provides a complementary means of analysing CMB observations instead 
of the angular power spectrum $C_{\ell}$. In principle, $C(\cos \theta)$ contains the same information as the angular power spectrum, but it provides an easier understanding of the anisotropic structures and may serve as a complementary means of spherical-harmonic analysis, which is the most commonly used method. Some authors (Smoot et al. 1992; Hinshaw et al. 1996; Kashlinsky et al. 2001; Copi et al. 2015; López-Corredoira \& Gabrielli 2013) have already attempted a direct determination of the anisotropies correlation function directly in angular space.

Several physical influences contribute to $C_{\ell}$, some preferentially at large angles (i.e. $\theta \gg 1-2^{\circ}$ ), others, such as baryon acoustic oscillations (BAO), predominantly on smaller scales (Meiksin et al. 1999; Seo \& Eisenstein 2005; Jeong \& Komatsu 2006; Crocce \& Scoccimarro 2006; Efstathiou et al. 2007; Padmanabhan \& White 2009). The 2013 release of the Planck temperature power spectrum (Planck Collaboration XII 2014) up to $\ell=30$ is shown in Fig. 1, along with two theoretical fits that we discuss shortly. In this figure, $D_{\ell} \equiv \ell(\ell+1) C_{\ell} / 2 \pi$. The well-known dichotomy between effects at large and small angles allows us to greatly simplify the calculation of $C_{\ell}$ for the purpose of this paper. At large angles, corresponding to $\ell \lesssim 30$, the dominant physical process producing the anisotropies is the Sachs-Wolfe (SW) effect (Sachs \& Wolfe 1967), representing metric perturbations associated with scalar fluctuations in the matter field. This effect relates the anisotropies observed in the temperature today to inhomogeneities of the metric fluctuation amplitude on the surface of last scattering. For the power-law spectrum of perturbations in Eq. (2), and assuming only SW, the angular power (Eq. (6)) reduces to the integral expression (Bond \& Efstathiou 1984; Hu \& Sugiyama 1995)

$C_{\ell}=N \int_{k_{\min }}^{\infty} k^{n-2} j_{\ell}^{2}\left(k c \Delta \tau_{\mathrm{dec}}\right) \mathrm{d} k$,

where $j_{\ell}$ is the spherical Bessel function of order $\ell$, and $c \Delta \tau_{\mathrm{dec}}$ is the comoving radius of the last scattering surface written in terms of the conformal time difference between $t_{0}$ and $t_{\mathrm{dec}}$. The normalization constant $N$ is typically determined by optimizing the fit to the data in Fig. 1, although in this paper we also show the impact of optimizing $N$ for $C(\cos \theta)$. We stress that the key difference between the conventional approach and the novel idea we are introducing here is the appearance of a non-zero lower limit, $k_{\min }$, to the integral in Eq. (7). As we see shortly, it is this $k_{\min }$ that accounts for the absence of CMB angular correlations at angles $\gtrsim 60^{\circ}$, representing a characteristic spatial scale apparently equal to $2 \pi$ times the gravitational radius $R_{\mathrm{h}}\left(t_{\mathrm{dec}}\right)=c / H\left(t_{\mathrm{dec}}\right)$ at decoupling.

From the WMAP and Planck observations, we infer that the power spectrum in Eq. (2) is very nearly scale free with $n \approx 1$ (Planck Collaboration XII 2014). Therefore, selecting this value in Eq. (7), and defining the variable

$u_{\min } \equiv k_{\min } c \Delta \tau_{\mathrm{dec}}$,

we may recast the expression for $C_{\ell}$ in the form

$C_{\ell}=N \int_{u_{\min }}^{\infty} \frac{j_{\ell}^{2}(u)}{u} \mathrm{~d} u$.

The constant $u_{\min }$ is $2 \pi$ times the number of proper maximum wavelengths $\lambda_{\max }$ (corresponding to $k_{\min }$ ) in the proper distance to the last scattering surface at $t_{\mathrm{dec}}$. Its value allows us to determine the angular size $\theta_{\max }$ of the largest fluctuation on this surface, using the expression

$u_{\min }=\frac{2 \pi}{\lambda_{\max }} a\left(t_{\mathrm{dec}}\right) c \Delta \tau_{\mathrm{dec}}$,

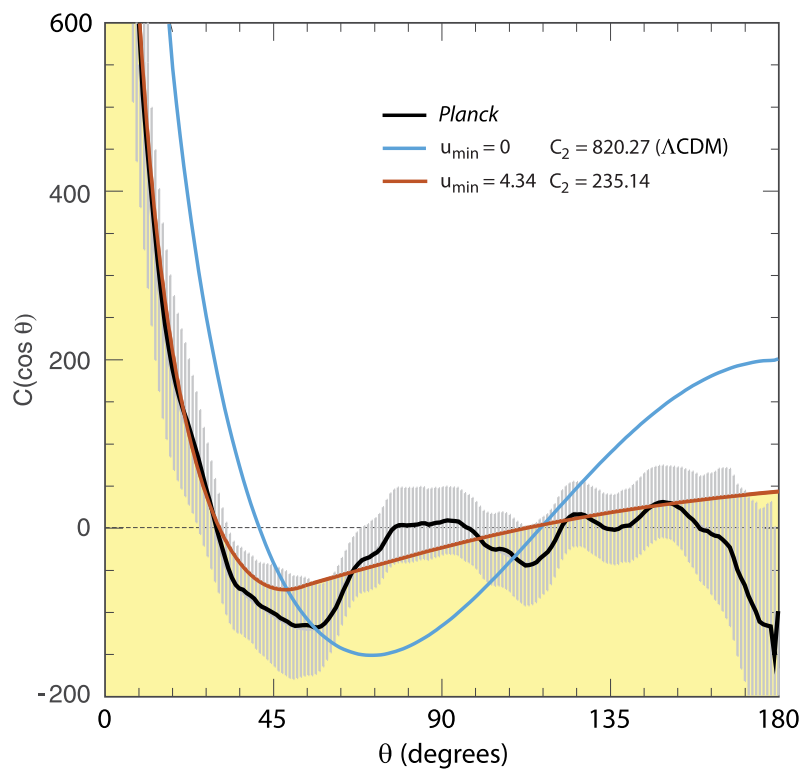

Fig. 2. Angular correlation function measured with Planck (dark solid curve) (Planck Collaboration XII 2014), and associated $1 \sigma$ errors (grey), compared with the prediction of the conventional inflationary $\Lambda \mathrm{CDM}$ (blue). In this model one assumes that $u_{\min }=0$ and then optimizes $C_{2}$ using the power spectrum in Fig. 1. The red curve shows the prediction of truncated inflation, or a non-inflationary cosmology, with an optimized lower limit $u_{\min }=4.34$ and $C_{2}=235.14$.

where $a(t)$ is the expansion factor. Therefore using the standard definition of the angular-diameter distance, this simply reduces to the form $\theta_{\max }=2 \pi / u_{\min }$.

\section{Discussion}

The CMB angular correlation function $C(\theta)$ based on the Planck 2013 release (Planck Collaboration XII 2014) has been calculated by averaging all temperature pairs in the sky separated by angles $\theta$ inside bins of size $1^{\circ}$. We used the component-separated CMB map resulting from the Needlet Internal Linear Combination (NILC) algorithm, downloaded from the Planck Legacy Archive $\left(\mathrm{PLA}^{1}\right)$. This map was smoothed to an angular resolution of $1^{\circ}$ and degraded to HEALPix ${ }^{2}$ (Górski et al. 2005) $N_{\text {side }}=64$ (pixel size $0.92^{\circ}$ ). In order to avoid foreground contamination from residual Galactic emission or point sources, we excluded from our analysis all pixels affected by the multiplication of the Commander, SEVEM, and SMICA masks, which keeps $67 \%$ of the sky; we did not take into account the NILC mask because it removes a significantly smaller fraction of the sky. The $1 \sigma$ error bars were computed through a comparison of the angular correlation function calculated for 200 randomly rotated maps based on the original distribution.

The calculated angular power $C_{\ell}$ for multipoles $2 \leq \ell \leq 30$ is shown for the conventional $\Lambda \mathrm{CDM}$ (blue curve) in Fig. 1, optimized to fit the Planck 2013 release. Also, for comparison, we show in this figure a fit for $u_{\min }=0$ (dashed) optimized using $C(\cos \theta)$ in Fig. 3 instead of the angular-power spectrum. In Fig. 1, the theoretical curves take into account only SW, ignoring the physical influences (such as BAO) that would dominate on small angular scales (i.e. at $\ell \gtrsim 30$ ). It is well known that the

\footnotetext{
1 http://www . cosmos.esa.int/web/planck/pla

2 https://healpix.jpl.nasa.gov
} 
latter depend, at most, only weakly on the cosmology, and are therefore degenerate among different models (Scott et al. 1995).

The fit for $u_{\min } \neq 0$ is optimized using $C(\cos \theta)$ in Fig. 2. For the $\Lambda \mathrm{CDM}$ (blue) curve, we followed the conventional approach of first fitting the temperature spectrum to determine the normalization constant $N$ in Eq. (9), which is then used together with Eq. (3) to produce the angular correlation function. It has been known since the early WMAP release (Spergel et al. 2003) that the $\Lambda \mathrm{CDM}$ curve is inconsistent with the measured $C(\cos \theta)$, but when viewed here in comparison with the curve corresponding to $u_{\text {min }} \neq 0$, its lack of adequate confirmation by the data is even more glaring. The red curve in Fig. 2 shows the best fit attainable with $u_{\text {min }} \neq 0$. Based on the Planck 2013 data release, we find an optimized value $u_{\min }=4.34 \pm 0.50$, corresponding to a maximum fluctuation size $\theta_{\max } \approx 83^{\circ}$ in the plane of the sky. For comparison with the value of $k_{\mathrm{c}}$ estimated earlier by Niarchou et al. (2004) using WMAP data, we determined for $\Lambda$ CDM (in which $z_{\mathrm{cmb}}=1080$ ) that this measurement of $u_{\min }$ corresponds to a maximum fluctuation size $\lambda_{\max } \sim 20 \mathrm{Mpc}$ at decoupling, which is about twice the value corresponding to their characteristic wave number $k_{\mathrm{c}}$. Of course, with the benefit of using the more precise Planck data, and our focus on the large-angle anomalies rather than the entire $\mathrm{CMB}$ power spectrum, we also conclude that a cut-off in $P(k)$ is favoured at a much higher level of significance than before, now in excess of $\sim 8 \sigma$.

This $\sigma=0.5$ error in the measurement of $u_{\text {min }}$ was obtained from a Monte Carlo analysis sampling the variation of $C(\cos \theta)$ within the measurement errors. The $C(\cos \theta)$ points are highly correlated, but our analysis circumvents this problem with the Monte Carlo procedure. First, we generated 100 mock CMBR catalogues [using standard cosmology with an angular function $C_{0}(\cos \theta)$ ] and we measured the two-point correlation $C_{i}(\cos \theta)$ in each case. From these, we obtained $\Delta C_{i}(\cos \theta) \equiv$ $C_{i}(\cos \theta)-C_{0}(\cos \theta)$, and then calculated $u_{\min , i}$ for $C(\cos \theta)=$ $C_{\text {Planck }}(\cos \theta)+\Delta C_{i}(\cos \theta)$ for each realization $i$. Next, we examined the distribution of $u_{\min , i}$ (which is roughly Gaussian) and determined its average value and the rms within which one finds 68 of the 100 values; this yields the quoted result $u_{\min }=$ $4.34 \pm 0.50$. This is not equivalent to a simple $\chi^{2}$ fitting with the correlated error bars shown in Figs. 2 and 3. Such a simple $\chi^{2}$ procedure would instead have given $u_{\min }=4.34_{-0.12}^{+0.10}$, i.e. a much smaller error for $u_{\min }$. This difference stems precisely from the fact that the $C(\cos \theta)$ points are correlated. Therefore the $68 \%$ confidence limit for this value of $u_{\text {min }}$ suggests that a theoretical best fit to the measured angular correlation function with $u_{\min }=0$ is rejected at $8.6 \sigma$.

From Fig. 2 we see that there are several good reasons for preferring the $u_{\min }=4.34$ fit over a model with $u_{\min }=0$ : (1) Planck has confirmed that $C(\cos \theta) \approx 0$ at angles $\theta \gtrsim 60^{\circ}-70^{\circ}$, in sharp contrast to the prediction of the conventional inflationary $\Lambda \mathrm{CDM}$; (2) the model with $u_{\min }=4.34$ correctly reproduces the minimum of $C(\cos \theta)$ at $\approx 45^{\circ}-50^{\circ}$; and (3) it actually fits the measured curve beyond $\sim 50^{\circ}$ quite well, which is fully consistent with the measurement errors.

Suppose we were to optimize $C_{2}$ with $u_{\min }=0$ based on the angular correlation data in Fig. 2, instead of the power spectrum in Fig. 1. This third case is shown as a dashed black curve in Fig. 3. Certainly, the fit to $C(\cos \theta)$ has improved, although there is still significant tension with theory at $\theta \lesssim 20^{\circ}$ and the best fit curve completely misses the minimum of $C(\cos \theta)$ at $\theta \sim 50^{\circ}$. In addition, with this optimized value of $C_{2}$ (i.e. $258.53 \mu \mathrm{K}^{2}$ ), the theoretical angular power spectrum is now a very poor fit to the measured $D_{\ell}$ s shown in Fig. 1. By far, the best fit to the angular

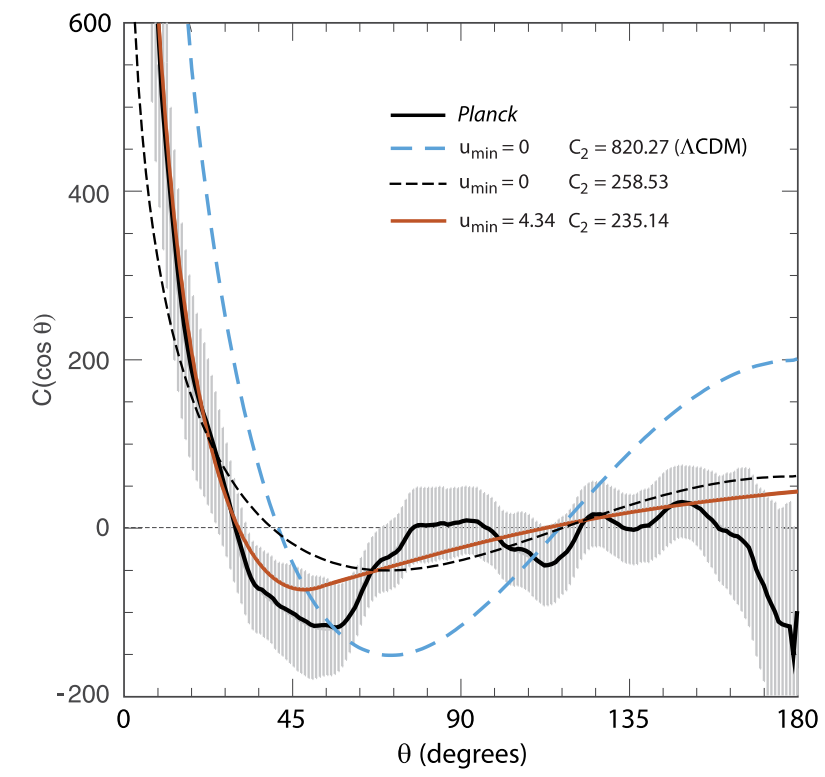

Fig. 3. Impact of $u_{\min }$ on the angular correlation function with truncated inflation, or a non-inflationary cosmology. The conventional $\Lambda \mathrm{CDM}$ curve corresponds to $u_{\min }=0$, i.e. an unconstrained inflationary power spectrum. The Planck measurement is shown as a black solid curve.

power spectrum and correlation function is realized when $u_{\min }=$ $4.34 \pm 0.50$.

\section{Conclusions}

The $S_{1 / 2}$ statistic (Spergel et al. 2003) has traditionally been used to categorize the degree to which the measured angular correlation at large angles is deficient compared to theoretical predictions. This quantity is basically an integral of $C(\theta)^{2}$ over angles from $\cos (\theta)=-1$ to $\cos (\theta)=1 / 2$. With Monte Carlo simulations, one can build a distribution of $S_{1 / 2}$ values, thereby assigning a probability that the observed angular correlation function could be drawn randomly as a result of cosmic variance from the predicted function. The $p$-values quoted earlier in this paper for conventional $\Lambda \mathrm{CDM}$ are estimated using this comparison.

Our analysis in this paper is superior to $S_{1 / 2}$ for several reasons. First, $S_{1 / 2}$ represents an integrated quantity, from $\theta \sim 60^{\circ}$ to $180^{\circ}$, designed to find a deficiency in signal. Second, it completely ignores the comparison between theory and observation at angles $\lesssim 60^{\circ}$, where the tension can be just a large as it is at $\theta \gtrsim 60^{\circ}$. Both of these limitations with $S_{1 / 2}$ make it an inferior statistic to use in this work compared to our approach of actually fitting the $C(\theta)$ data by optimizing the value of $u_{\min }$. The fact that the conventional inflationary paradigm is disfavoured by these data in comparison to a model with $k_{\min }>0$ is supported by both approaches. But the optimization carried out in this paper goes significantly beyond this level by demonstrating that $k_{\text {min }}=0$ is ruled out at a confidence level of $\sim 8 \sigma$.

A $k>k_{\min }$ constraint on $P(k)$ is inconsistent with purely slow-roll inflationary cosmology, which instead predicts that fluctuations would have exited and re-entered the horizon prior to decoupling, resulting in $k_{\min }=0$ and strong correlations at all angles. On the other hand, such a result is fully consistent with the predictions of the $R_{\mathrm{h}}=c t$ universe (Melia 2007, 2013; Melia \& Shevchuk 2012), in which fluctuations might have emerged 
near the Planck scale, equal to the Hubble radius $R_{\mathrm{h}}$ at the Planck time. The $k_{\min }$ might therefore correspond to the horizon size at the surface of last scattering, since only fluctuations with $\lambda_{\max } \sim 2 \pi R_{\mathrm{h}}$ would have continued to grow towards $t_{\mathrm{dec}}$.

The fact that the measured CMB angular correlation function strongly favours modifying conventional inflation, or eliminating it all together, is an important validation of other recent results showing similar trends (Wei et al. 2016). Looking forward to the next generation of observations and simulations, the introduction of a non-zero $k_{\min }$ should be quite straightforward to implement. The introduction of a $k_{\min }$ will not affect many other kinds of observation. The optimized value we found here corresponds to a maximum fluctuation angle in the sky of about $83^{\circ}$. As such, this will have no impact on the optimization of the power spectrum, since $k_{\min }$ affects only the far-left (i.e. $\ell \sim 1-10$ ) portion of $D_{\ell}$ in Fig. 1. By comparison, the first acoustic peak in the power spectrum is centred at $\ell \sim 200$ corresponding to an angle of $\sim 1^{\circ}$. For similar reasons, such a large maximum fluctuation angle is unlikely to affect other measurements. At least for now, the focus with $k_{\min }$ should be on refining the measurement of the angular correlation function of the CMB.

From a simulational point of view, however, we have so far ignored the integrated Sachs Wolfe (ISW) effect, which arises owing to the passage of light from the surface of last scattering to our location (Bond \& Efstathiou 1984). The ISW is not negligible, although the early-time contribution is typically combined with SW at last scattering and is incorporated into Eq. (7). The late-time ISW is smaller, but nonetheless present, hence a complete accounting of the impact of $k_{\min }$ will eventually need to include its influence. We do not expect our results to change substantially.

Acknowledgements. We are very grateful to Ricardo Génova-Santos for help calculating the angular correlation function using the Planck 2013 data release. F.M acknowledges partial support from the Chinese State Administration of Foreign Experts Affairs under grant GDJ20120491013. M.L.C. was supported by grant AYA2015-66506-P of the Spanish Ministry of Economy and Competitiveness.

\section{References}

Bennett, C. L., Hill, R. S., Hinshaw, G., et al. 2003, ApJS, 148, 97 Bennett, C. L., Hill, R. S., Hinshaw, G., et al. 2011, ApJS, 192, 17 Bennett, C. L., Larson, D., Weiland, J. L. et al. 2013, ApJS, 208, 20

Bond, J. R., \& Efstathiou, G. 1984, ApJ, 285, L45

Brandenberger, R. H. 1994, IJMP-A, 9, 2117

Contaldi, C. R., Peloso, M., Kofman, L., \& Linde, A. 2003, JCAP, 7, 002

Copi, C. J., Huterer, D., Schwarz, D. J., \& Starkman, G. D. 2007, PRD, 75, 023507

Copi, C. J., Huterer, D., Schwarz, D. J., \& Starkman, G. D. 2015, MNRAS, 451, 2978

Crocce, M., \& Scoccimarro, R. 2006, PRD, 73, 063520

Destri, C., de Vega, H. J., \& Sanchez, N. G. 2010, PRD, 81, 063520

Efstathiou, D. J. 2004, MNRAS, 348, 885

Eisenstein, D. J., Seo, H.-J., \& White, M. 2007, ApJ, 664, 660

Górski, K., Hivon, E., Banday, A. J., et al. 2005, ApJ, 622, 759

Gruppuso, A., Kitazawa N., Mandolesi, N., P. N., Sagnotti, A. 2016, PDU, 11, 68 Guth, A. H. 1981, PRD, 23, 347

Hinshaw, G., Banday, A. J., Bennett, C. L., et al. 1996, ApJ, 464, L25

Hu, W., \& Sugiyama, N. 1995, ApJ, 444, 489

Jeong, D., \& Komatsu, E. 2006, ApJ, 651, 619

Kashlinsky, A., Hernández-Monteagudo, A., \& Atrio-Barandela, F. 2001, ApJ, 557, L1

Kim, J., \& Naselsky, P. 2011, ApJ, 739, 79

Linde, A. 1982, PLB, 108, 389

López-Corredoira, M., \& Gabrielli, A. 2013, Physica A, 392, 474

Meiksin, A., White, M., \& Peacock, J. A. 1999, MNRAS, 304, 851

Melia, F. 2007, MNRAS, 382, 1917

Melia, F. 2013, A\&A, 553, A76

Melia, F. 2014, A\&A, 561, A80

Melia, F. 2016, Front. Phys., 11, 118901

Melia, F. 2017, Front. Phys., 12, 129802

Melia, F., \& Shevchuk, A. 2012, MNRAS, 419, 2579

Niarchou, A., Jaffe, A. H. \& Pogosian, L. 2004, PRD, 69, 063515

Padmanabhan, N., \& White, M. 2009, PRD, 80, 063508

Planck Collaboration XII. 2014, A\&A, 571, A12

Planck Collaboration XXIII. 2014, A\&A 571, A23

Planck Collaboration XVI. 2016, A\&A, 594, A16

Sachs, R. K., \& Wolfe, A. M. 1967, ApJ, 147, 73

Scott, D., Silk, J., \& White, M. 1995, Science, 268, 829

Seo, H.-J., \& Eisenstein, D. J. 2005, ApJ, 633, 575

Smoot, G. F., Bennett, C. L., Kogut, A., et al. 1992, ApJ, 396, L1

Spergel, D. N., Verde, L., Peiris, H.V, et al. 2003, ApJS, 148, 175

Wei, J.-J., Wu, X., \& Melia, F. 2016, MNRAS, 463, 1144

Wright, E. L., Bennett, C. L., Gorski, K., et al. 1996, ApJ, 464, L21 